
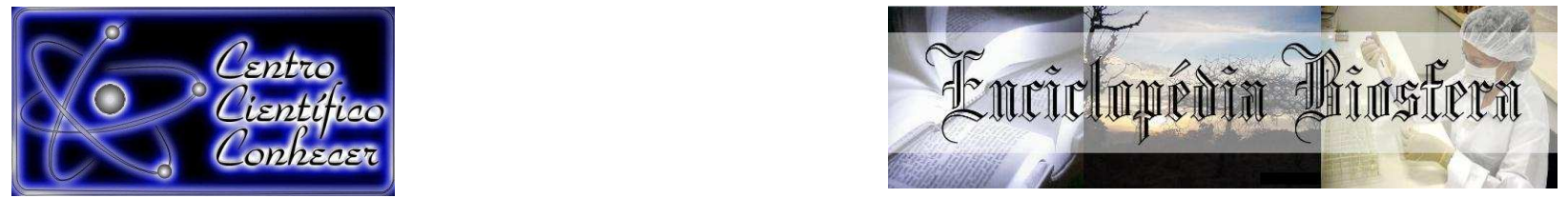

\title{
ESTUDO CIENCIOMÉTRICO DAS PRODUÇÕES CIENTÍFICAS SOBRE SAÚDE DA MULHER
}

\author{
Carolina Correia Bilotti ${ }^{1}$, Carolina Arnaut dos Santos ${ }^{1}$, Mirian Ueda Yamaguchi ${ }^{2}$, Ely \\ Mitie Massuda ${ }^{2}$, Marcelo Picinin Bernuci ${ }^{2}$ \\ ${ }^{1}$ Mestrandas do Programa de Pós-Graduação em Promoção da Saúde, UniCesumar. \\ Maringá PR Brasil. \\ ${ }^{2}$ Professores Doutores do Programa de Pós-Graduação em Promoção da Saúde, \\ UniCesumar e pesquisadores do Instituto Cesumar de Ciência, Tecnologia e Inovação - \\ ICETI. Maringá PR Brasil. \\ Autor correspondente: elymitie.m@gmail.com
}

Recebido em: 03/10/2016 - Aprovado em: 21/11/2016 - Publicado em: 05/12/2016 DOI: 10.18677/EnciBio_2016B_133

\section{RESUMO}

Objetivou-se avaliar o estado atual da produção científica no campo da saúde da mulher, para determinar a sua tendência e padrão após o período de implementação da Política Nacional de Atenção Integral a Saúde da Mulher (PNAISM). Por meio de estudo cienciométrico, a triagem dos artigos foi realizada em uma base de dados nacional (Scielo) e outra internacional (Pubmed) utilizando palavras-chave relevantes para o tema. Após aplicação dos critérios de inclusão foram selecionados 126 artigos, sendo que 114 referem-se aos eixos da PNAISM e 12 relacionam-se a outras áreas temáticas. A Atenção Obstétrica e Neonatal corresponde a $51 \%$ das produções científicas, sendo o eixo com maior número de publicações, seguido de $16,6 \%$ artigos sobre planejamento familiar e $11,4 \%$ sobre câncer de mama e colo. A análise permitiu compreender que é necessária a ampliação de pesquisas que abordem as diversidades das mulheres brasileiras, com intuito de subsidiar a criação de políticas de saúde aplicáveis no contexto nacional.

PALAVRAS-CHAVE: Brasil; Políticas Públicas.Saúde da mulher

\section{SCIENTOMETRIC STUDY OF SCIENTIFIC PRODUCTIONS ON WOMEN'S HEALTH}

\begin{abstract}
We aimed to evaluate the current status of scientific production in the field of women's health, to determine its trend and pattern after the implementation of National Policy for Integral Attention to Women's Health (PNAISM). Using relevant keywords, we searched one national database (Scielo) and one international database (Pubmed) to locate the papers published in the field by scienciometric study. After application of the inclusion criteria were selected 126 articles, and 114 refer to the axes of PNAISM and 12 relates to other subject areas. The Obstetric and Neonatal corresponds to $51 \%$ of the scientific production, the axis with the highest number of publications followed $16.6 \%$ publications on family planning and $11.4 \%$ of breast and colon cancer. The analysis allowed us to understand that it is necessary the expansion of studies that address the diversity of Brazilian women, which will allow the creation of relevant health policies throughout the national context.
\end{abstract}

KEYWORDS: women's health, Brazil, public policies

ENCICLOPÉDIA BIOSFERA, Centro Científico Conhecer - Goiânia, v.13 n.24; p.1445 


\section{INTRODUÇÃO}

A Constituição Federal do Brasil de 1988, delimitando as responsabilidades de todas as esferas de governo e não apenas da União e direito do trabalhador segurado, determinou a saúde como um direito universal, passando a se vincular às políticas sociais e econômicas (CORDONI, 2006; BRASIL, 201). O Sistema Único de Saúde - SUS, conhecido também como a política pública brasileira de saúde, implantado no ano de 1990, apresenta como uma de suas atribuições a elaboração de políticas e a execução das ações em saúde (PAULUS \& CORDONI, 2006).

O artigo 197 da Constituição Federal de 1988 apresenta a saúde pública de forma composta e descentralizada, sendo de responsabilidade das três esferas governamentais fazendo-se necessária a elaboração de políticas públicas de saúde nesses espaços (BRASIL, 2012). O surgimento de políticas de saúde dirigidas a grupos específicos ocorre com o passar dos anos. Em 2002 aprovam-se as Políticas Nacionais de Saúde da Pessoa Portadora de Deficiência, de Atenção à Saúde dos Povos Indígenas e de Redução da Morbimortalidade por Acidentes e Violências e, em 2004, as Políticas Nacionais de Humanização do SUS, de Saúde do Trabalhador e de Atenção Integral à Saúde da Mulher (BRASIL, 2011).

Segundo dados da Pesquisa Nacional por Amostra de Domicílios no Brasil, a participação das mulheres na população é de $51,4 \%$, com exceção na região Norte cuja parcela corresponde a $49,9 \%$ da população (BRASIL, 2015b). A razão de gênero aponta que para cada grupo de 100 mulheres na população, existem 96 homens em 2010 no Brasil (BRASIL, 2014). As mulheres em sua grande maioria vivem mais que os homens devido às vantagens biológicas e comportamentais, o que não significa que essa longevidade seja saudável, uma vez que existem condições vividas por elas com impactos negativos que podem acarretar riscos à saúde e requerem cuidados como, por exemplo, a gestação e o parto (OMS, 2011).

Os Sistemas de Saúde em diversos países negligenciam as mulheres, apesar de serem elas as que mais contribuem para a atenção em saúde por meio dos cuidados primários que realizam aos familiares (OMS, 2011). No Brasil, quando se analisam as questões relativas à saúde, observa-se uma implantação gradativa de políticas nacionais de saúde direcionadas à saúde da mulher, tendo seu início nas primeiras décadas do século XX.

Em 2004 foi publicada a Política Nacional de Atenção Integral a Saúde da Mulher (PNAISM). O documento aponta que apesar das importantes diferenças do perfil epidemiológico da população feminina nas distintas regiões do país, consideradas as diferenças socioeconômicas e culturais assim como as condições de acesso aos serviços e ações de saúde, não se aborda as questões de heterogeneidade da população em profundidade (BRASIL, 2004).

Ainda no contexto de políticas direcionadas às mulheres, surgiu em 2005, o Plano Nacional de Políticas para as Mulheres (PNPM). As ações do Plano foram traçadas considerando quatro linhas de atuação principais, tendo como objetivo promover a melhoria da saúde das mulheres brasileiras, garantir o acesso aos meios e serviços de promoção, prevenção, assistência e recuperação da saúde em todo território nacional, os direitos sexuais e direitos reprodutivos das mulheres e contribuir para a redução da morbidade e mortalidade feminina no Brasil (BRASIL, 2005).

Para melhor compreensão das publicações sobre políticas de saúde direcionadas às mulheres, nesse artigo, analisa-se a produção científica vinculada às Políticas Públicas de Saúde direcionadas às mulheres no Brasil por meio do estudo cienciométrico. Os estudos cienciométricos possibilitam a análise quantitativa 
e qualitativa da produção científica em um campo específico, especialmente em saúde (YAO et al.2014; ZANG et al., 2014), sendo importante para direcionar as novas estratégias e diretrizes.

\section{MATERIAL E MÉTODOS}

A metodologia foi baseada na abordagem cienciométrica da literatura científica nacional e internacional sobre as Políticas Nacionais de Atenção à Saúde da Mulher. A pesquisa foi realizada nas bases de dados United States Nacional Library of Medicine (PubMed) disponível em http://www.ncbi.nlm.nih.gov/pubmed, Scientific Eletronic Library Online (Scielo), disponível em http://www.scielo.br/, Literatura Latino-Americana e do Caribe em Ciências da Saúde (Lilacs), disponível em http://lilacs.bvsalud.org/ e Centro Latino-Americano e do Caribe de Informação em Ciências da Saúde, disponível no website http://bvsalud.org/.

Efetivou-se a busca dos artigos no mês de março de 2016 empregando-se os termos "política saúde mulher" e seu correspondente em inglês "policy woman health acrescido da palavra Brazil. Foram incluídas publicações datadas no período compreendido entre o ano 1970 e março de 2016.

Os critérios de inclusão utilizados foram: a) abordar tema relacionado à Saúde da Mulher; b) referir às Políticas Públicas Brasileiras; e c) ser redigido em língua portuguesa e/ou inglesa.

Após leitura dos títulos e resumos, os artigos incluídos no estudo foram agrupados em 12 áreas temáticas abordadas na Política Nacional de Atenção Integral a Saúde da Mulher (PNAISM): 1) Planejamento Familiar; 2) Atenção Obstétrica e Neonatal; 3) Violência Doméstica e Sexual; 4) DST/HIV/AIDS; 5) Câncer de Mama e Colo; 6) Saúde Mental; 7) Climatério; 8) Terceira Idade; 9) Mulher Negra; 10) Saúde das Trabalhadoras do Campo e Cidade; 11) Mulher Indígena; e 12) Saúde das Presidiárias. Os artigos que abordaram temas complementares foram agrupados em eixos comuns.

A análise de dados seguiu abordagem descritiva.

\section{RESULTADOS E DISCUSSÃO}

Inicialmente, localizaram-se 306 artigos assim distribuídos: 244 na base de dados PubMed; e 62 na SciELO. Os títulos e resumos foram examinados, após a exclusão dos artigos duplicados e também aqueles que não se encaixaram nos critérios de inclusão. Dessa forma, 126 artigos forma retidos (Figura 1).

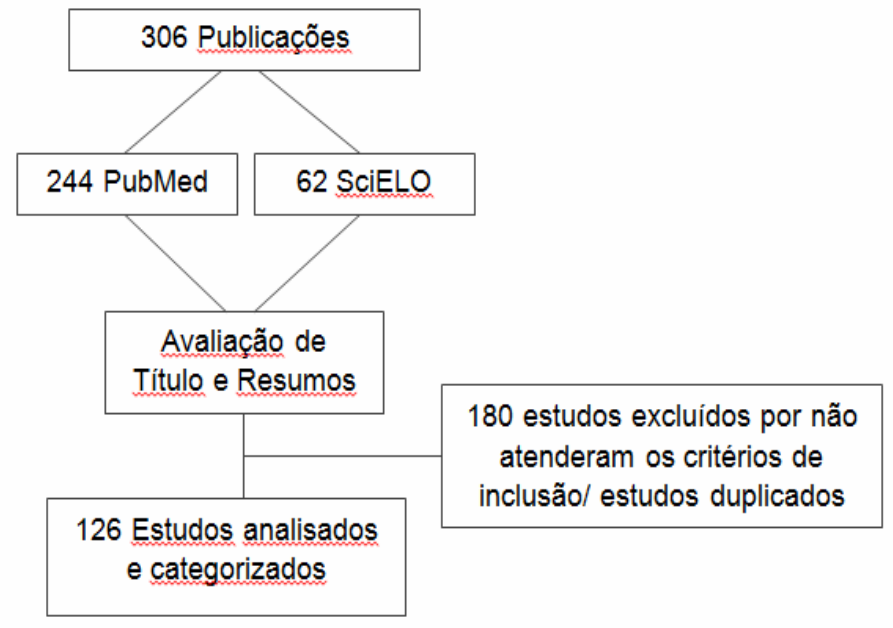

FIGURA 1. Diagrama representativo da seleção de estudos que constituíram a amostra do presente trabalho. 
Dos 126 estudos selecionados, 114 referem-se aos eixos da PNAISM e 12 relaciona-se a outras áreas temáticas. O perfil das publicações que integram os eixos temáticos da PNAISM: a Atenção Obstétrica e Neonatal somou 59 artigos, correspondendo a $51 \%$ das produções científicas, sendo o eixo com maior número de publicações; seguido de 19 (16,6\%) publicações sobre planejamento familiar; e $13(11,4 \%)$ sobre câncer de mama e colo. Os eixos temáticos "mulher negra, saúde das trabalhadoras do campo e cidade, bem como saúde das presidiárias" apresentaram apenas uma publicação, cada um deles. Saúde mental e mulher indígena configuram como os eixos temáticos sem publicações identificadas (Figura 2).

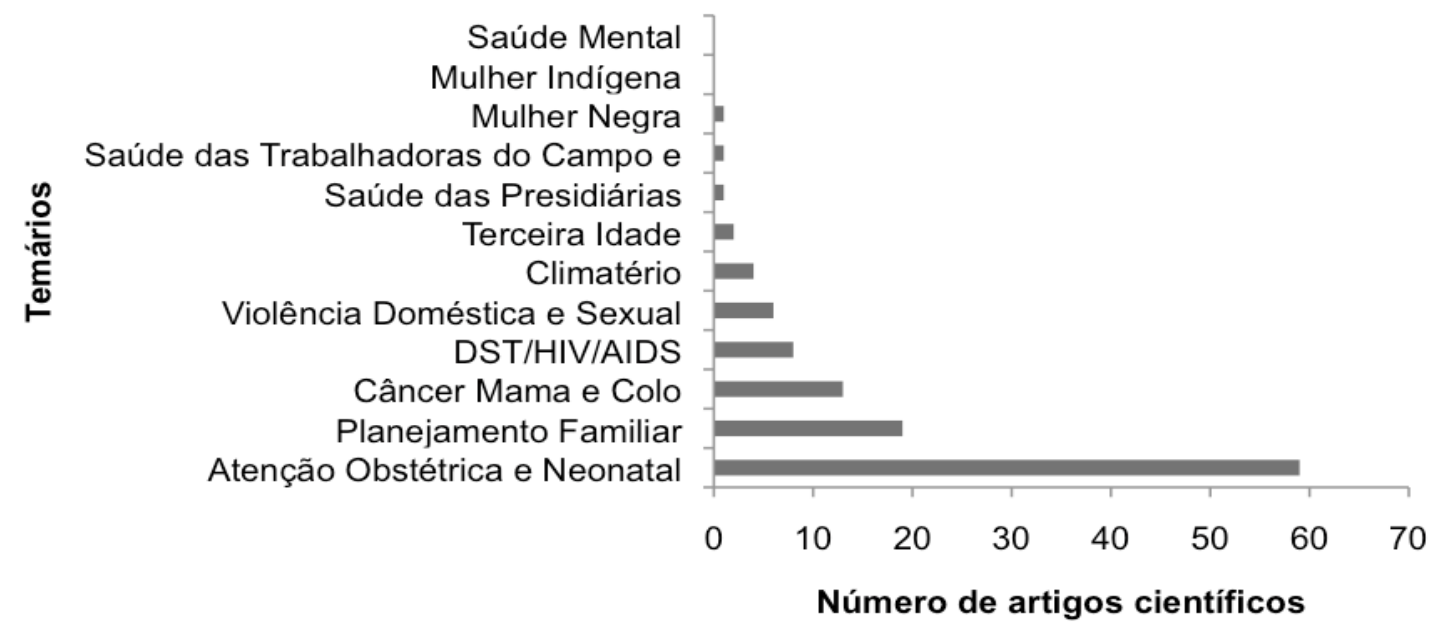

FIGURA 2. Perfil da produção científica relacionada aos eixos temáticos da Política Nacional de Atenção Integral à Saúde da Mulher (PNAISM).

Verifica-se que as publicações estão concentradas na atenção obstétrica e neonatal. Se somadas aquelas referentes ao Planejamento Familiar, atingem 67,6\% do total. As demais temáticas da PNAISM não possuem número expressivo de estudos, podendo refletir-se na assistência as mulheres que se enquadram nessas áreas específicas, o que também pode significar situação de vulnerabilidade dessa população.

O anseio em serem reconhecidas para além dos aspectos biológicos foi demonstrado por mulheres de assentamento rural submetidas a vulnerabilidades, dada à condição de trabalho braçal e exposição às intempéries da natureza. Denotase a necessidade de ampliação e adequação ao contexto da realidade das ações que subsidiam a elaboração das políticas públicas (EBLING et al., 2015). Quando se aborda a população indígena, essa necessidade de adequação ao contexto é evidenciada, uma vez que os conhecimentos disponibilizados pelas políticas públicas, muitas vezes, acabam passando por um processo de indigenização, tomando um caminho diferente daquilo que foi proposto a nível geral (FERREIRA, 2013). Ouvir e dialogar com povos culturalmente distintos, como no caso das mulheres indígenas, é uma necessidade para que as políticas públicas de saúde estejam em conformidade com o princípio da equidade (FERREIRA, 2013a).

Embora se reconheça a importância da rede de relações que se tecem entre as famílias e as mulheres em situação de prisão, a dificuldade de acesso às políticas públicas de saúde e à assistência social é um relato comum entre os familiares de 
mulheres encarceradas, fragilizando a credibilidade quanto às ações do Estado (PEREIRA, 2016). Outro aspecto evidenciado é a ausência de estudos no eixo saúde mental. Esta constatação é importante pois evidencia um alerta para pesquisadores, uma vez que a presença de transtorno mentais em mulheres é algo frequente e muitas vezes não diagnosticado por profissionais da área (RENNÓ, 2012).

Nos últimos anos, a saúde da mulher está em debate e vem conquistando espaço nas políticas públicas. No entanto, mesmo com representação em vários conselhos como, por exemplo, no Conselho Nacional de Saúde (CNS), ainda é rara a inclusão da temática da saúde da mulher em pautas de debates, o que prejudica em vários aspectos os ajustes necessários nas políticas para que haja a inclusão das diversidades especificas das mulheres brasileiras (COSTA, 2009).

A Figura 3 apresenta o perfil das 12 produções científicas relacionadas aos eixos temáticos complementares da PNAISM. A maior porcentagem $(75 \%)$ das publicações é atribuída as Políticas de Saúde para Mulher e os demais $25 \%$ se distribuem igualmente entre os temas tabagismo, consumo de álcool e obesidade. Para esses eixos, o Plano Nacional de Políticas para as Mulheres (PNPM), reforça o processo de consolidação e amadurecimento das políticas para as mulheres, evidencia a participação social, sobretudo das mulheres, como sendo constitutiva em todas as fases do ciclo das políticas públicas (BRASIL, 2013).

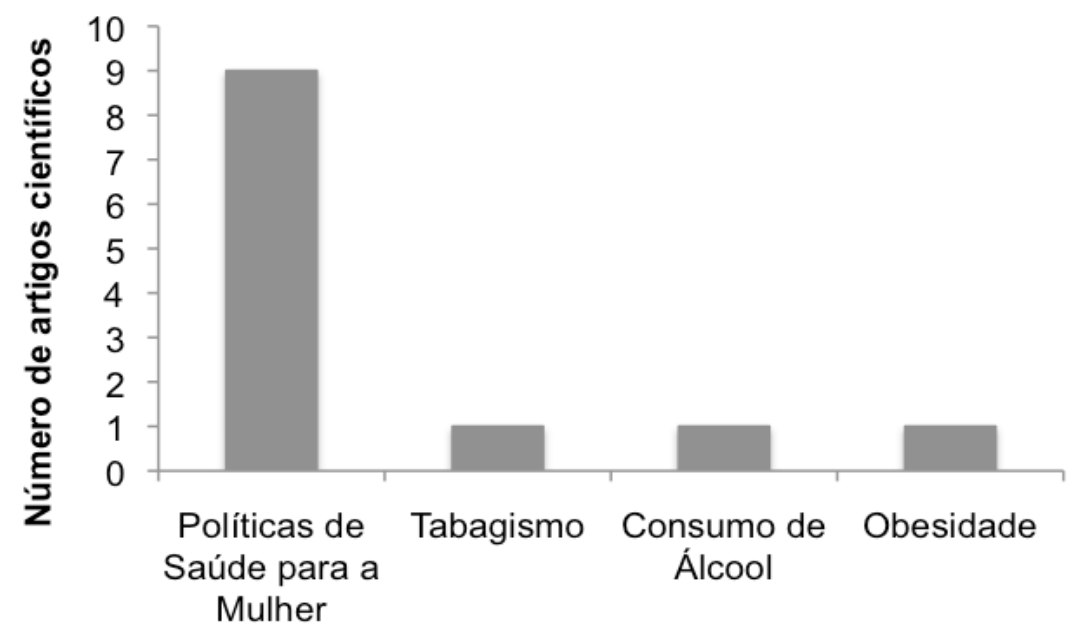

Temários

FIGURA 3. Perfil da produção científica relacionada aos eixos temáticos complementares da Política Nacional de Atenção Integral a Saúde da Mulher (PNAISM).

Constatou-se um número pequeno de pesquisas ligadas a hábitos de consumo prejudiciais à saúde e fatores preponderantes para o desenvolvimento das principais doenças crônicas não transmissíveis, como consumo de álcool e tabaco. O Ministério da Saúde entende as ações de prevenção e promoção da saúde como primordiais (BRASIL, 2015). Para tanto propõe que as intervenções em saúde ampliem seu escopo, tomando como objeto os problemas e as necessidades de saúde, seus determinantes e condicionantes. A Política Nacional de Promoção da Saúde de 2006 evidencia como tema prioritário de ações, o enfretamento ao álcool e 
tabaco, bem como alimentação saudável equilibrada. Para tanto incentiva a articulação de ações com corresponsabilização e autonomia da população, que visem à redução do tabaco e uso abusivo do álcool (BRASIL, 2015a).

Não foram observadas publicações direcionadas às mulheres portadoras de necessidades especiais. No próprio PNAISM, o tema surge apenas no campo das diretrizes citadas entre parênteses, apontando que o desafio das políticas públicas de saúde para as mulheres ainda é a integralidade (SANTOS et al., 2013). No que se refere à saúde da mulher e as políticas públicas de saúde direcionadas a essa população, é necessário aprofundamento das questões não se baseando apenas no modelo biomédico de assistência, mas também nas peculiaridades e nas necessidades individuais e comunitárias desse grupo. Espera-se com esse estudo que, novos campos da saúde da mulher sejam abordados em pesquisas nos próximos anos.

\section{CONSIDERAÇÕES FINAIS}

As pesquisas direcionadas à saúde da mulher no Brasil se concentram na Atenção Obstétrica e Neonatal e o Planejamento Familiar, portanto, em âmbito cujo papel feminino se limita a essas áreas, reproduzindo a condição histórica na sociedade. No entanto, compreender a saúde da mulher no sentido amplo significa considerar além dos aspectos ligados a essas esferas que são igualmente importantes, abranger também questões contempladas pela própria PNAISM, quais sejam: violência doméstica e sexual; DST/HIV/AIDS; câncer de mama e colo; saúde mental; climatério; terceira idade; mulher negra; saúde das trabalhadoras do campo e cidade; mulher indígena; saúde das presidiárias, perpassando as diversidades sociodemográficas, econômicas, culturais e étnicas existentes na sociedade brasileira.

Recomenda-se a ampliação de estudos que abordem as diversidades das mulheres brasileiras, os quais poderão subsidiar as políticas de saúde aplicáveis em todo o contexto nacional.

\section{REFERÊNCIAS}

BRASIL. Conselho Nacional de Secretários de Saúde. A gestão do SUS/ Conselho Nacional de Secretários de Saúde. - Brasília: CONASS, 2015.

BRASIL. Constituição da República Federativa do Brasil texto constitucional promulgado em 5 de outubro de 1988, com as alterações adotadas pelas Emendas Constitucionais nos 1/1992 a 68/2011, pelo Decreto Legislativo no 186/2008 e pelas Emendas Constitucionais de Revisão nos 1 a 6/1994. 35.ed. Brasília : Câmara dos Deputados, Edições Câmara, 2012.

BRASIL. Ministério da Saúde. Secretaria de Atenção à Saúde. Departamento de Ações Programáticas Estratégicas. Política Nacional de Atenção integral à Saúde da Mulher: princípios e diretrizes, Brasília, 2004.

BRASIL. Ministério da Saúde. Secretaria de Vigilância em Saúde. Secretaria de Atenção à Saúde. Política Nacional de Promoção da Saúde: PNPS: revisão da Portaria MS/GM no 687, de 30 de março de 2006/ Ministério da Saúde, Secretaria de Vigilância em Saúde, Secretaria de Atenção à Saúde. - Brasília: Ministério da Saúde, 2015a. 
BRASIL. Ministério da Saúde. Secretaria-Executiva. Subsecretaria de Assuntos Administrativos. SUS: a saúde do Brasil, Brasília: Editora do Ministério da Saúde, 2011.

BRASIL. Ministério do Planejamento, Orçamento e Gestão Instituto Brasileiro de Geografia e Estatística - IBGE. Sistema Nacional de Informações de Gênero Estatísticas de Gênero. Diretoria de Pesquisas Coordenação de População e Indicadores Sociais Estudos e Pesquisas Informação Demográfica e Socioeconômica. Uma análise dos resultados do Censo Demográfico 2010, n.33, Rio de Janeiro, 2014.

Brasil. Ministério do Planejamento, Orçamento e Gestão. Instituto Brasileiro de Geografia e Estatística - IBGE. Pesquisa nacional por amostra de domicílios: síntese de indicadores 2013. Coordenação de Trabalho e Rendimento. 2. ed. Rio de Janeiro, 2015b.

BRASIL. Presidência da República. Secretaria de Políticas para as Mulheres. Plano Nacional de Políticas para as Mulheres. Atualização. Brasília: Secretaria de Políticas para as Mulheres, 2013.

BRASIL. Presidência da República. Secretaria Especial de Políticas para as Mulheres. Plano Nacional de Políticas para as Mulheres, Brasília, 2005.

COSTA, A. M. Participação social na conquista das políticas de saúde para mulheres no Brasil. Ciência e Saúde Coletiva, v. 14, n. 4, p. 1073-83, 2009. Disponível em: <http://www.scielo.br/scielo.php?script=sci_arttext\&pid=S141381232009000400014> doi: 10.1590/S1413-81232009000400014

EBLING, S. B.; FALKEMBACH, E. F.; NASCIMENTO, L. A.; SILVA, M. M.; SILVA, S. de O.; MINUSSI, P. S.; As mulheres e suas lidas: compreensões acerca de trabalho e saúde. Trabalho, Educação e Saúde, v. 13, n. 3, p. 581-596, 2015. Disponível em: <http://www.scielo.br/scielo.php?script=sci_arttext\&pid=S1981$77462015000300581 \&$ Ing=pt\&nrm=iso\&tIng=en>. doi: 10.1590/1981-7746-sip00065.

FERREIRA, L. O. A emergência da medicina tradicional indígena no campo das políticas públicas. História, Ciências, Saúde-Manguinhos, v. 20, n. 1, p. 203-219, 2013. Disponível em: <http://www.scielo.br/pdf/hcsm/v20n1/11.pdf>. doi: $10.1590 /$ S0104-59702013000100011

FERREIRA, L. O. Saúde e relações de gênero: uma reflexão sobre os desafios para a implantação de políticas públicas de atenção à saúde da mulher indígena. Ciência e Saúde Coletiva, v. 18, n. 4, p. 1151-1159, 2013. Disponível em < http://www.scielo.br/scielo.php?script=sci_arttext\&pid=\$1413-81232013000400028 > doi: 10.1590/S1413-81232013000400028

OMS - Organização Mundial da Saúde. Mulheres e Saúde Evidências de Hoje Agenda de Amanhã. 2011. <Disponível em: http://www.who.int/ageing/mulheres_saude.pdf> 
PAULUS JÚNIOR, A.; CORDONI, L. Políticas públicas de saúde no Brasil. Revista Espaço para a Saúde, v.8, n.1, p. 13-19, 2006.

PEREIRA, E. L. Famílias de mulheres presas, promoção da saúde e acesso às políticas sociais no Distrito Federal, Brasil. Ciência e Saúde Coletiva, v.21, n.7, p.2123-2134, 2016. Disponível em < http://www.scielo.br/scielo.php?pid=S141381232016000702123\&script=sci_abstract\&tlng=pt>. doi: 10.1590/141381232015217.16792015.

RENNÓ JR, J. ; MARQUE, R. de, LOBO, H. R., CAVALSAN, J. P., SILVA, A. G. da. Saúde Mental da Mulher: transtornos psiquiátricos relacionados ao ciclo reprodutivo. Revista Debates em Psiquiatria, v. 12, n.2, p. 6-11, 2012.

SANTOS, L. F. de M. dos, BENTO, P. A. de S., TELLES, A. C., RODRIGUES, R. F., XAVIER, R. B. Mulheres com deficiência: reflexões sobre a trajetória das políticas públicas de saúde. Revista de Enfermagem UFPE on Line, v.7, n.7, p. 4775-4781 2013. <www.revista.ufpe.br/revistaenfermagem/index.php/revista/article/download/4384/66 32 > doi: 10.5205/reuol.4656-38001-2-SM.0707201326

YAO, Q., CHEN K., YAO L., LYU P.H, YANG, T,A., LUO. F., CHEN, S.Q., HE L.Y., LIU, Z,Y. Scientometric trends and knowledge maps of global health systems research. Health Research Policy Systems, v.12, n.26, p.1-20, 2014; Disponível em: < https://health-policy-systems.biomedcentral.com/articles/10.1186/1478-450512-26 > doi: 10.1186/1478-4505-12-26

ZHANG, Y., DIAO, T., WANG, L. Quantative evalution of translational medicine based on scientometric analysis and information extraction. Clinical and Translational Science Awards, v.7, n.6, p. 465-469, 2014. Disponível em: <https://www.ncbi.nlm.nih.gov/pubmed/25079489> doi: 10.1111/cts.12193 